\title{
Benchmarking of Narrowband LPWA Physical Layer Ranging Technologies
}

\author{
Florian Wolf*\|, Kévin Le Déroff*, Sébastien de Rivaz*, Nicolas Deparis*, François Dehmas* and Jean-Pierre Cances" \\ * CEA-Leti Minatec Campus, 17 rue des Martyrs, 38054 Grenoble Cedex 09, France \\ Email: \{florian.wolf, kevin.lederoff, sebastien.derivaz, nicolas.deparis, francois.dehmas\}@cea.fr \\ "| Université de Limoges, CNRS, XLIM, UMR 7252, 87000 Limoges, France \\ Email: \{florian.wolf, jean-pierre.cances\}@xlim.fr
}

\begin{abstract}
Accurate radio signal based geolocalization for Low Power Wide Area networks is a key-enabler to various Internet of Things applications. However, localization with narrowband signals remains challenging in multipath environments. Sequential coherent multi-channel ranging improves temporal resolution while being compatible with narrowband transmissions. New radio chipsets integrate proprietary ranging functions. This paper compares a proof-of-concept implementation for coherent multi-channel ranging with narrowband signals to the Time-of-Flight ranging function of the LoRa $2.4 \mathrm{GHz}$ radio chip. Benchmarking results for different configurations and propagation scenarios are discussed, illustrating the precision scalability of multi-channel ranging.
\end{abstract}

Index Terms-Multi-channel, frequency hopping, LoRa 2.4 GHz SX1280, LPWA network localization, range estimation

\section{INTRODUCTION}

Low Power Wide Area (LPWA) networks offer long-range, low data rate wireless connectivity to a large variety of devices such as environmental or personal health monitoring devices. Many of these so-called Internet of Things (IoT) applications can be enhanced through geolocation information. Georeferencing the wireless transmitted alert of a vital-sign patch will improve guiding a rescuing team rapidly to a person suffering a heart stroke [1]. Extracting geolocation based on data-carrying LPWA signals meets low power, low complexity and low cost requirements and is hence a promising alternative to obtain position on Global Navigation Satellite System (GNSS)-denied devices. However, obtaining precise location with LPWA signals remains challenging. Firstly, LPWA technologies utilize narrowband modulation schemes to achieve long-range communication with low transmit power. Consequently and in contrast to ultra-wideband radio localization, LPWA radio signals do not offer sufficient temporal resolution for precise ranging [2]. Secondly, IoT applications, such as the vital-sign patches, face multipath propagation and blockage of the radio signal in urban environments. These scenarios impede accurate and unbiased range estimation.

Narrowband-IoT (NB-IoT), LoRa and Sigfox are LPWA technologies offering geolocation information with different characteristics. NB-IoT signals typically occupy a single Long Term Evolution (LTE) resource block of $180 \mathrm{kHz}$ bandwidth [3]. Localization of NB-IoT devices by Time-of-Flight (ToF) measurements attains ranging errors inferior to $140 \mathrm{~m}$ in $90 \%$ of the cases [4]. The Sigfox radio technology achieves long-range communication in urban environments through ultra-narrowband signals. Hence, time based ranging with signal bandwidths down to $100 \mathrm{~Hz}$ is unfeasible and geolocation is derived from received signal strength (RSS) measurements combined with fingerprinting [5], achieving $500 \mathrm{~m}$ positioning error. LoRa radios attain similar receiver sensitivity levels through spread spectrum modulation. Urban LoRa networks in the $868 \mathrm{MHz}$ Industrial Scientific Medical (ISM) band achieve $200 \mathrm{~m}$ errors based on a Time-Difference-of-Arrival (TDoA) method and $250 \mathrm{kHz}$ chirp signals [6], requiring however specific location-enabled gateways and ToF is not supported. A new radio chip (SX1280) in the $2.4 \mathrm{GHz}$ ISM band offers bandwidths up to $1.6 \mathrm{MHz}$ and has a build-in ranging engine [7]. Existing work [8]-[10] only provides few benchmarking of the ranging feature. Coherent multi-channel ranging allows improving temporal resolution through the aggregation of sequentially transmitted narrowband signals [11]. This technique is compatible with LPWA constraints and offers advanced geolocalization. Various studies [12]-[14] have demonstrated the performance gain compared to ToF ranging. An implementation for LPWA networks [11] is hereafter denoted Coherent Ranging On Narrowband Enabled Networks (CRONEN).

The present work compares LPWA adapted multi-channel ranging with CRONEN [11], to the LoRa $2.4 \mathrm{GHz}$ SX1280 ranging feature [7], in the absence of a comparable ranging system in the $868 \mathrm{MHz}$ ISM band. Focus lies on ranging accuracy and precision.

The contributions of this paper are twofold:

- A Python programming based hardware-software architecture with direct physical layer access for rapid benchmarking of the SX1280 ToF ranging feature.

- Benchmarking and comparison between ranging performances of multi-channel ranging, i.e. CRONEN and the SX1280 in different scenarios.

This paper is organized as follows. Section II introduces the SX1280 ranging feature and the LPWA multi-channel CRONEN transceiver testbed. Section III outlines the methodology for ranging performance comparison. Benchmarking results are given in Section IV and Section V concludes this work. 


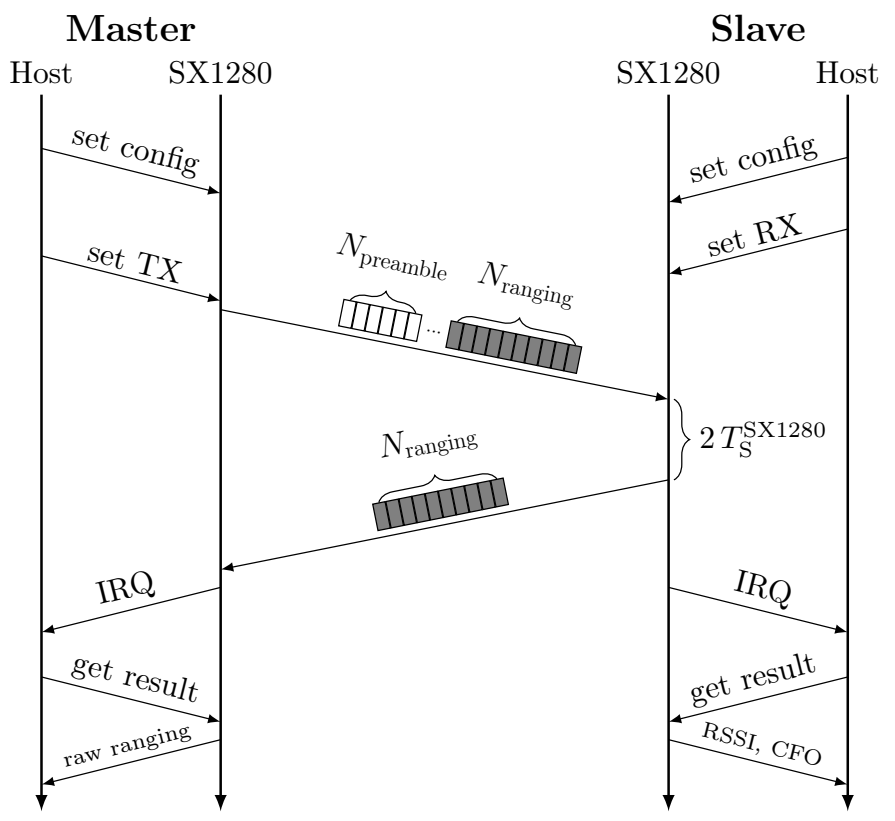

Fig. 1. LoRa 2.4 GHz SX1280 Time-of-Flight (ToF) ranging protocol.

\section{LPWA PhySiCAL LAYER RANGING TECHNOLOGIES}

\section{A. LoRa $2.4 \mathrm{GHz}$ SX1280 Time-of-Flight (ToF) ranging}

1) Physical layer and ranging feature: The LoRa $2.4 \mathrm{GHz}$ SX1280 radio chip [7] is an evolution of the sub-GHz LoRa chirp spread spectrum radio, offering higher bandwidth configurations and an integrated ToF ranging engine.

A LoRa symbol is defined as linear frequency chirp of bandwidth $B W$ grouping $S F$ bits into a symbol of duration $T_{\mathrm{S}}^{\mathrm{SX} 1280}=2^{S F} / B W$.

The ToF ranging principle is depicted in Fig. 1. A master and a slave node each comprising a SX1280 as well as a host controller perform a two-way packet exchange [7], [15].

Required steps are as follows:

1) Both - Configuration: The host controllers set radio parameters such as carrier frequency, transmit power $P_{\mathrm{TX}}$, bandwidth $B W$, spreading factor $S F$, number of preamble symbols $N_{\text {preamble }}$ and number of ranging symbols $N_{\text {ranging. }}$.

2) Slave - Set RX: The node that is assigned the slave role is set into ranging reception mode waiting for a ranging request from the master.

3) Master - Set TX: After the slave has been set to RX, the master can send its ranging packet. Upon reception by the slave, the latter checks identification, estimates clock offset, aligns itself to the master clock and responds with a ranging response packet.

4) Both - Ranging finished: Interrupts (IRQ) indicate the results of the ranging packet exchange, i.e. failed communication or successful range estimation. In this latter case, host controllers can retrieve the results:

- Master: Raw ranging result.
- Slave: RSS and Carrier Frequency Offset (CFO).

Various calibration and correction steps need to be performed on the raw ranging result in order to obtain precise and accurate range estimates [15], [16]. These procedures are detailed in Section IV. The receiver has a maximum sensitivity of $-132 \mathrm{dBm}$ [7].

2) Python based benchmarking architecture: Classical approaches often dedicate a microcontroller for controlling the radio chipset. While offering advantages in terms of integration and real time operation, this approach requires rather long software development $(\mathrm{C} / \mathrm{C}++$ language $)$ and is limited in flexibility, which is crucial when benchmarking a new radio physical layer.

In this work, an architecture has been chosen that allows the direct configuration and controlling of the SX1280 radio chipset $^{1}$ with a Raspberry Pi3 Model $\mathrm{B}+$ and the high-level programming language Python. The setup is depicted in Fig. 2 and Fig. 3 and offers the following advantages:

- Rapid prototyping.

- Flexible configuration of the Medium Access Layer (MAC) and direct access to the Physical (PHY) layer.

- Storage of radio physical layer outputs in time series databases (i.e. InfluxDB) and quasi real-time visualization (i.e. Grafana).

- Connectivity for a GNSS module in order to obtain ground truth to establish ranging errors in outdoor benchmarking. Connectivity via IP (e.g. Ethernet, WiFi or LTE) for remote controlling and monitoring of the setup.

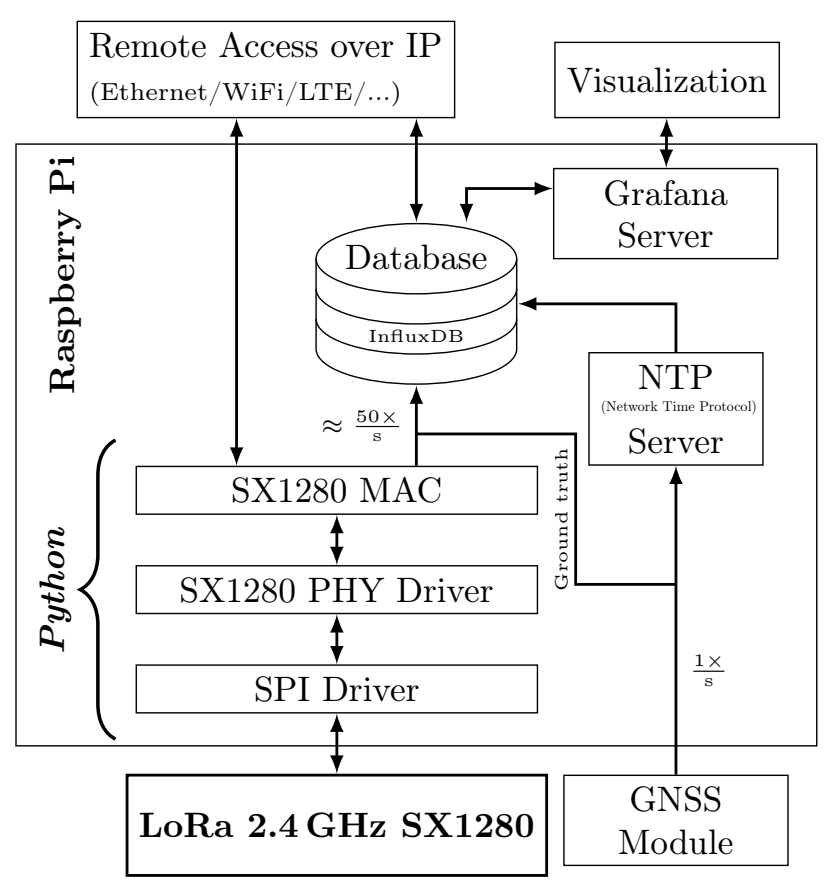

Fig. 2. Raspberry Pi and Python based benchmarking architecture for the LoRa $2.4 \mathrm{GHz}$ SX1280 Time-of-Flight ranging feature.

${ }^{1}$ Evaluation Board SX1280DVK1ZHP, E394V02A. 


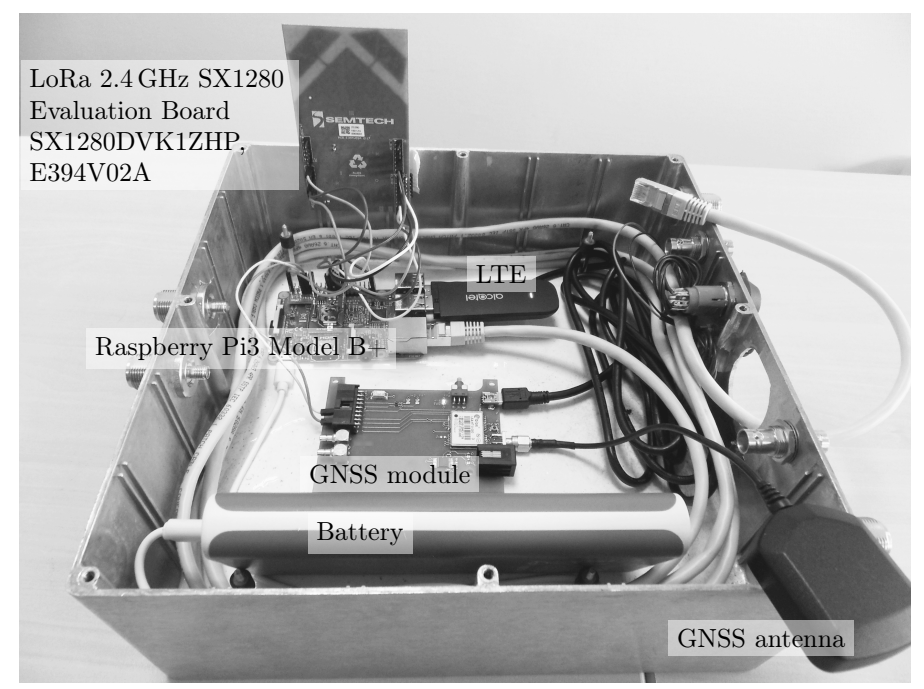

Fig. 3. LoRa $2.4 \mathrm{GHz}$ SX1280 Time-of-Flight ranging platform. Experiments are performed with external, off-board antennas.

\section{B. CRONEN - Sequential multi-channel ranging}

Sequential multi-channel ranging, i.e. CRONEN (see Fig. 4) samples the channel transfer function $H(f)$ at $f=c \Delta f+f_{\mathrm{R}}$ with $c \in[0, C-1]$ channels of spacing $\Delta f$ and carrier frequency $f_{\mathrm{R}}$. Applying the inverse discrete Fourier transform (iDFT) results in the sampled version of the channel impulse response $h(t)$ [13]. Range estimation is performed through detecting the first path in the reconstructed channel impulse response. For such a processing scheme, range resolution is conditioned by the virtual bandwidth $B W_{\text {virt }}$ and is given by $\Delta R=c_{0} /(2(C-1) \Delta f)=c_{0} /\left(2 B W_{\text {virt }}\right)$ and maximum unambiguous range $R_{\max }=c_{0} /(2 \Delta f)$ [17]. Details on this method can be found in [11], [13].

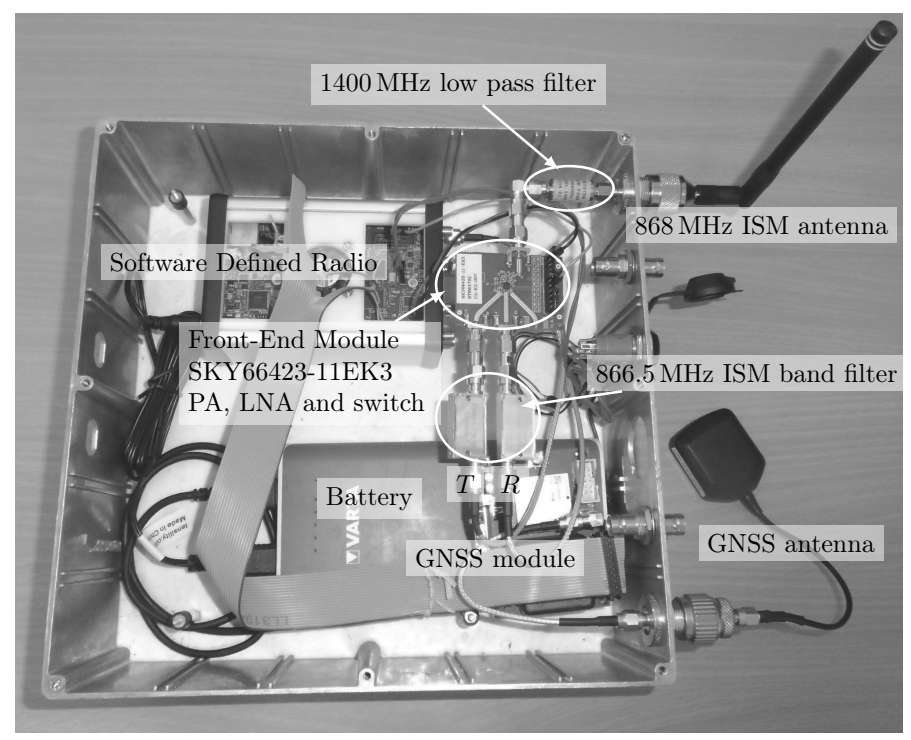

Fig. 4. CRONEN transceiver testbed comprising a Software Defined Radio (SDR), radio frequency components, a GNSS module and a power supply.
In the present work, CRONEN transmits a Binary-Phase-Shift-Keying (BPSK) preamble of chip rate $R_{\mathrm{c}}=10 \mathrm{kchip} / \mathrm{s} \equiv B W_{\mathrm{sym}}=10 \mathrm{kHz} \quad$ following a Barker code of chip length $N_{\mathrm{c}}=7$ chip. The channel transfer function is sampled at $C=16$ frequencies with channel spacing $\Delta f=200 \mathrm{kHz}$ in the $f_{\mathrm{R}}=868 \mathrm{MHz}$ ISM band. Hence the multi-channel protocol occupies $B W_{\text {virt }}=3 \mathrm{MHz}$. A receiver sensitivity of about $-123 \mathrm{dBm}$ assures a $5 \mathrm{~m}$ ranging precision in an Additive White Gaussian Noise (AWGN) channel [11].

\section{Methodology for COMPARISON}

LPWA networks underlie energy consumption and regulation constraints and hence a fair comparison of associated ranging technologies can be given for equal time-on-air $T_{\text {air }}$ and equal received power ${ }^{2} P_{\mathrm{RX}}$, which yields equal ranging symbol energy

$$
E_{\mathrm{S}, \text { ranging }}^{X}=P_{\mathrm{RX}} T_{\text {air }}^{X}=P_{\mathrm{RX}} \begin{cases}\frac{2^{S F}}{B W_{\text {ranging }}} & X=\mathrm{SX} 1280, \\ \frac{N_{c}}{B W_{\mathrm{sym}}} C & X=\text { CRONEN } .\end{cases}
$$

Furthermore, receiver sensitivity with noise spectral density $N_{0} \approx-174 \mathrm{dBm} / \mathrm{Hz}$

$$
S=\left(E_{\mathrm{s}} / N_{0}\right)_{\min }+N_{0}-10 \log _{10}\left(T_{\mathrm{S}}\right)+N F,
$$

in Table I and hence coverage are comparable, neglecting different noise figures $N F$ and the difference in path loss $(8.83 \mathrm{~dB})$ for the $868 \mathrm{MHz}$ and the $2.4 \mathrm{GHz}$ ISM band.

For the configuration in Table $\mathrm{I}, E_{\mathrm{S} \text {,ranging }}^{\mathrm{SX} 1280}=E_{\mathrm{S} \text {,ranging }}^{\mathrm{CRNN}}$ and consequently the ranging Cramer Rao lower bound (CRLB)

$$
\sigma_{\tau, \mathrm{CRLB}}^{X}=1 / \sqrt{4 \pi^{2}\left(E_{\mathrm{S}, \text { ranging }}^{X} / N_{0}\right) B W_{\mathrm{rms}}^{2}},
$$

only depends on the root-mean-square (RMS) bandwidth

$$
B W_{\mathrm{rms}}=\sqrt{\int_{-\infty}^{\infty} f^{2}\left|S_{0}(f)\right|^{2} d f / \int_{-\infty}^{\infty}\left|S_{0}(f)\right|^{2} d f}
$$

\begin{tabular}{|c|c|c|}
\hline & LoRa 2.4 GHz SX1280 & CRONEN \\
\hline $\begin{array}{l}\text { Receiver } \\
\text { sensitivity } S\end{array}$ & -117 dBm [7] & $-123 \mathrm{dBm}[11]$ \\
\hline $\begin{array}{l}\text { Symbol } \\
\text { time } T_{\mathrm{S}}\end{array}$ & $\begin{aligned} \frac{2^{S F}}{B W} & =\frac{2^{10}}{1625.00 \mathrm{kHz}} \\
& =0.63 \mathrm{~ms}\end{aligned}$ & $\begin{aligned} \frac{N_{\mathrm{c}}}{B W_{\mathrm{sym}}} & =\frac{7}{10 \mathrm{kHz}} \\
& =0.7 \mathrm{~ms}\end{aligned}$ \\
\hline $\begin{array}{l}\text { One-way } \\
\text { ranging } \\
\text { duration } \\
T_{\text {ranging }}\end{array}$ & $\begin{aligned} T_{\mathrm{S}} N_{\text {ranging }} & =0.63 \mathrm{~ms} \cdot 18 \\
& =11.34 \mathrm{~ms}\end{aligned}$ & $\begin{aligned} T_{\mathrm{S}} C & =0.7 \mathrm{~ms} \cdot 16 \\
& =11.2 \mathrm{~ms}\end{aligned}$ \\
\hline
\end{tabular}

TABLE I

LPWA RANGING PLATFORM CONFIGURATIONS

${ }^{2}$ Also equal transmit power $P_{\mathrm{TX}}$ if same path loss, i.e. same frequency band. 


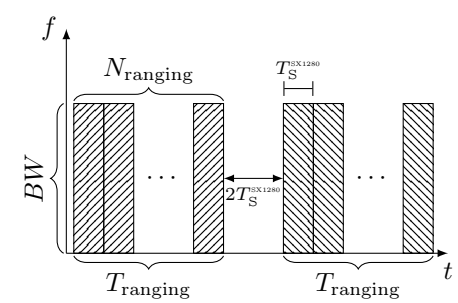

(a) $\mathrm{SX} 1280$

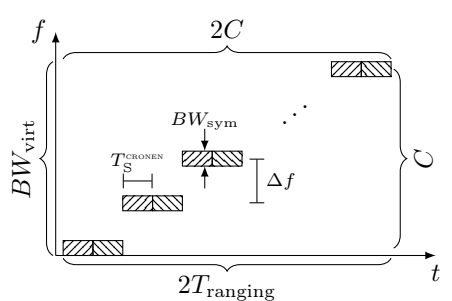

(b) CRONEN
Fig. 5. Spectrum occupation for the two-way ranging protocols.

where $S_{0}$ represents the spectrum of the baseband waveform $s_{0}$. The RMS bandwidth for SX1280 and CRONEN are given by ${ }^{3}$

$$
\begin{aligned}
B W_{\mathrm{rms}}^{\mathrm{SX} 1280} & =B W / \sqrt{12}, \\
B W_{\mathrm{rms}}^{\mathrm{CRONEN}} & =\sqrt{\frac{B W_{\mathrm{sym}}^{2}+\Delta f^{2}\left(C^{2}-1\right)}{12}},
\end{aligned}
$$

with the advantage for CRONEN that ranging precision becomes scalable ${ }^{4}$ without degrading receiver sensitivity through increased bandwidth. CRONEN occupies at each time less spectrum and Fig. 5 shows that analog-to-digital conversion only requires sampling rates of $B W_{\mathrm{sym}}$ compared to $B W$ for LoRa, which further reduces power consumption for a CRONEN implementation.

The following benchmarking is performed, unless stated otherwise, with the configuration of Table I and transmit power is set on both platforms to $12.5 \mathrm{dBm}$. For receive power levels above the sensitivity level, benchmarking allows characterizing the ranging functionality in terms of robustness against multipath propagation. Moreover, with this choice, the LPWA critical characteristic of energy consumption of both platforms is comparable.

Neglecting antenna radiation patterns, a fair comparison can be established with this methodology.

\section{RANING BENCHMARKING}

The SX1280 benchmarking section aims at confirming performance announcements of the application note [15], explaining various calibration and correction procedures and benchmarking the SX1280 ranging feature in different real propagation scenarios. The second part focuses on comparison of CRONEN ranging performances to those of the SX1280.

\section{A. SX1280 ranging feature evaluation}

1) SX1280 cabled setup: Master and slave are connected via a short cable $(<1 \mathrm{~m})$ and an $80 \mathrm{~dB}$ attenuation. The range error histograms for more than 1000 ranging exchanges per configuration are illustrated in Fig. 6. Increasing bandwidth by a factor 4 , improves the ranging precision proportionally, which is conform to the application note and the CRLB. The residual, systematic biases are subtracted from the measurements.

\footnotetext{
${ }^{3}$ Assuming a rectangular shape of the spectrum.

${ }^{4}$ Channel spacing has to be smaller than channel coherence bandwidth for proper $H(f)$ reconstruction.
}

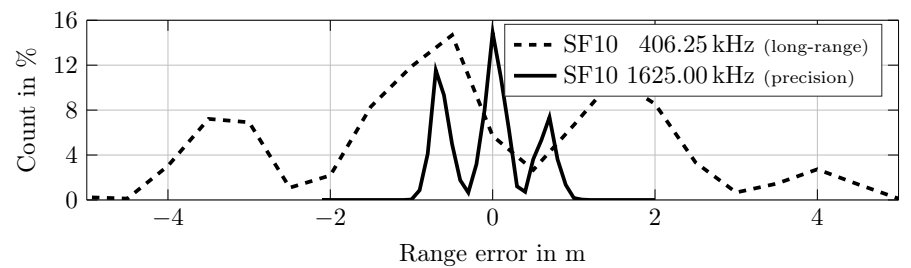

Fig. 6. LoRa $2.4 \mathrm{GHz} \mathrm{SX} 1280$ range precision in a cabled AWGN channel for the long-range $(\sigma=2.1 \mathrm{~m})$ and precision $(\sigma=0.5 \mathrm{~m})$ configuration.

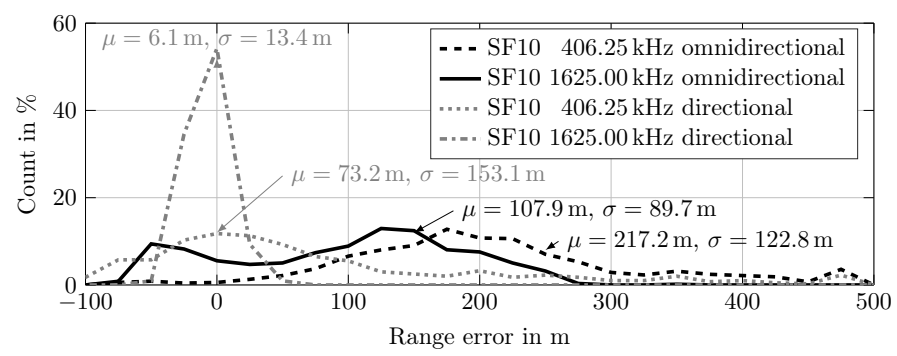

Fig. 7. LoRa $2.4 \mathrm{GHz} \mathrm{SX} 1280$ ranging error for a ground based setup over $550 \mathrm{~m}$ with omnidirectional and directional antenna on the master.

2) SX1280 ground-ground outdoor: Fig. 9 depicts master (M) and slave (S), which are setup within Line of Sight $(\mathrm{LoS})$ in a straight road at distance of $550 \mathrm{~m}$ with omnidirectional antennas at a height of $1.5 \mathrm{~m}$. Passing vehicles temporarily obstruct the LoS in the open industrial area.

Fig. 7 shows the ranging error histograms for the long-range $(406.25 \mathrm{kHz})$ and the precision $(1625.00 \mathrm{kHz})$ configuration. Large biases $(\approx 200 \mathrm{~m})$ are observed which can be interpreted as a multipath reflection from a large building $b_{1}$ facade approximately $100 \mathrm{~m}$ behind the master-slave line. The precision configuration is capable of resolving the attenuated direct ground path in some cases. Utilizing a directive antenna ${ }^{5}$ on the master, pointing towards the slave in order to suppress the multipath from the building $b_{1}$ behind the master significantly reduces the range biases. Although transmit power has been reduced to compensate the directional antenna gain, ranging precision equally improves for the precision configuration.

\section{B. Comparison of CRONEN to the LoRa $2.4 \mathrm{GHz}$ SX1280}

Based on the methodology for comparison given in Section III, SX1280 and CRONEN ranging performances are compared in a real outdoor scenario. Fig. 9 shows the ground truth positions at which measurements are performed.

The raw range estimations with omnidirectional antennas for both platforms are illustrated in Fig. 8. A total of 50000 and 3500 measurements have been collected for SX1280 and CRONEN respectively.

SX1280 two-way ranging exchanges fail for ranges $\gtrsim 500 \mathrm{~m}$ as depicted in Fig. 8a. For the CRONEN solution, the range ambiguity $R_{\max }$ is not resolved in this work. Consequently, an error $\pm R_{\max } / 2$ signifies no range information, i.e. in the $N o$

\footnotetext{
${ }^{5} \mathrm{WiFi}$ sector antenna $19 \mathrm{dBi}, 60^{\circ} \mathrm{H}, 6^{\circ} \mathrm{V}$.
} 


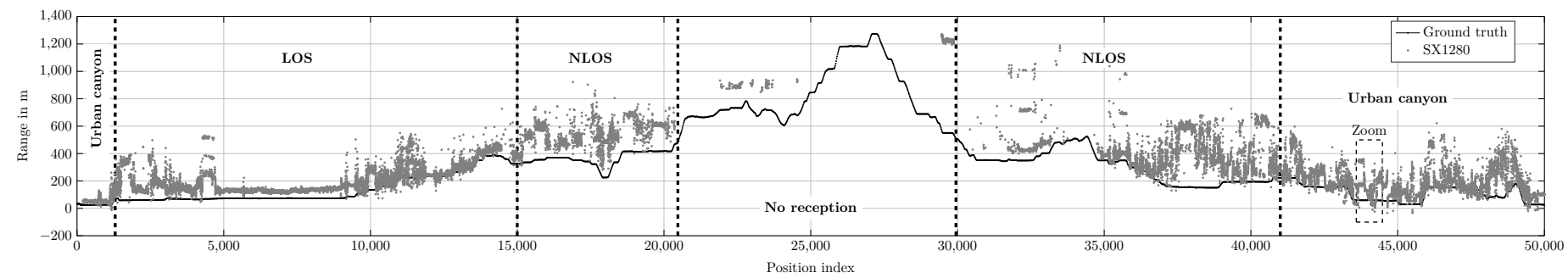

(a) LoRa $2.4 \mathrm{GHz}$ SX1280 raw range estimates.

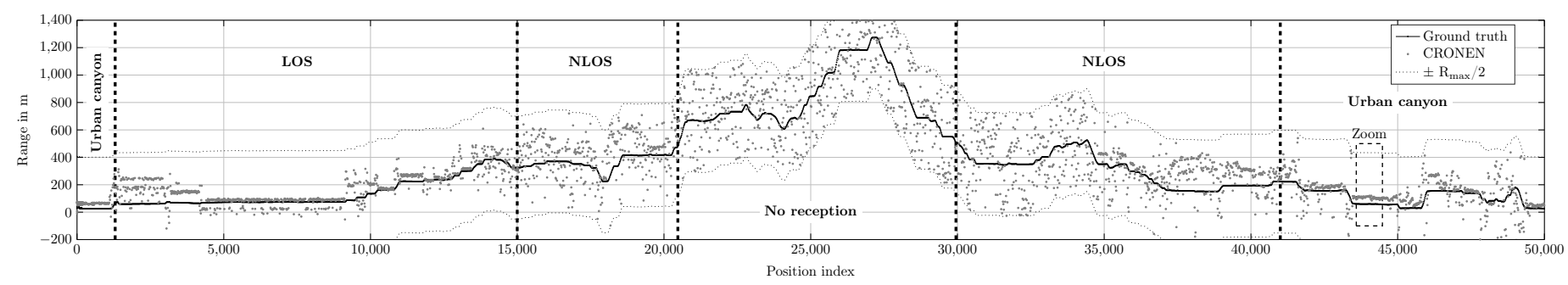

(b) CRONEN raw range estimates.

Fig. 8. LoRa $2.4 \mathrm{GHz}$ SX1280 and CRONEN raw range estimates for the real outdoor scenario in Fig. 9.

reception zone. Failed range estimation is due to the building $b_{1}$ blocking the LoS.

1) Line-of-Sight (LoS) outdoor: Fig. 10 depicts the range error for the LoS scenario. For a moving mobile node (car, speed $<36 \mathrm{~km} / \mathrm{h}$ ), CRONEN range estimation fails as channel phase changes between successive measurements, which is reflected by an almost uniform distributed range error in $\left[0, R_{\max } / 2\right]$. For the $\mathrm{SX} 1280$ ranging errors are inferior to $40 \mathrm{~m}$ in $50 \%$ of the moving cases. For stationary measurements CRONEN outperforms the SX1280 by a factor 2, which is coherent with the RMS bandwidth ratio $B W_{\mathrm{rms}}^{\mathrm{CRONEN}} / B W_{\mathrm{rms}}^{\mathrm{SX} 1280}$.

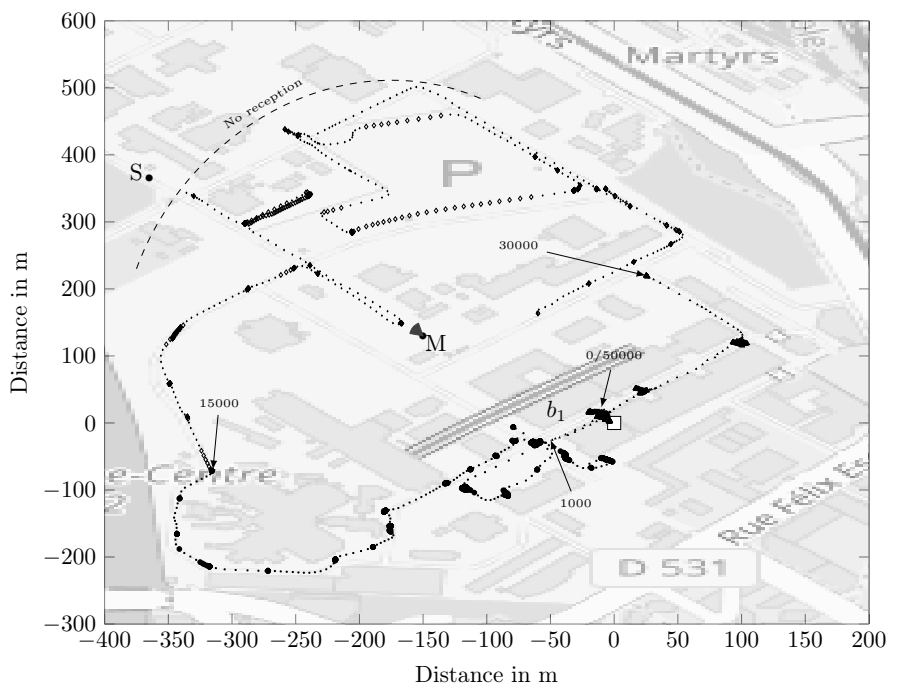

Fig. 9. Ground truth positions for the comparison of LoRa $2.4 \mathrm{GHz}$ SX1280 and CRONEN. Base station ( $\square$ ) at height $26 \mathrm{~m}$ and mobile node at height $1.5 \mathrm{~m}$. Mobile node in $\operatorname{LoS}(\bullet), \operatorname{NLoS}(\diamond)$ and urban canyon $(\triangle)$ scenario. In bold are stationary position indexes.
2) Non Line-of-Sight (NLoS): Raw range estimations in the NLoS scenario are not exploitable. However, methods to detect such a NLoS scenario can be applied. An obstruction and NLoS propagation detection method [18] based on the Received Signal Strength (RSS) can be applied to SX1280 measurements. CRONEN offers the advantage that the available estimation of the channel impulse response allows applying more sophisticated NLoS detection methods, i.e. [19].

3) Urban canyon: A zoom on raw range estimation results in the urban canyon is depicted in Fig. 11. For the position index $43680-43830$, the mobile node was strictly stationary. The SX1280 range estimates alternate between two distinct values, which can be explained by small-scale fading due to dynamics in the environment causing multipath components to vary in amplitude. The SX1280 ToF based range estimation acquires either one of these two paths present in this scenario. For the position index $43965-44280$, the mobile node has been displaced very slowly $(\approx 1 \mathrm{~cm} / \mathrm{s})$ and the $\mathrm{SX} 1280$ range measurements vary in a sweep-like manner over more than $100 \mathrm{~m}$. Meanwhile CRONEN range estimates show less variability as well as a smaller bias.

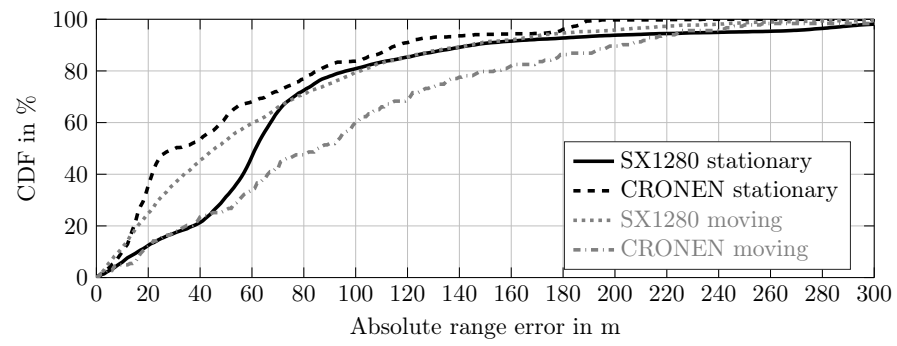

Fig. 10. LoRa 2.4 GHz SX1280 and CRONEN range error Cumulative Distribution Function (CDF) for the LoS scenario. 


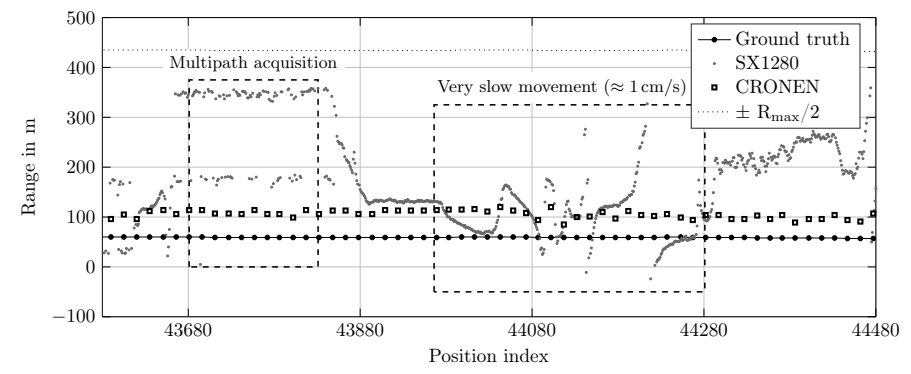

Fig. 11. LoRa $2.4 \mathrm{GHz}$ SX1280 and CRONEN raw range estimates in the urban canyon (zoom).

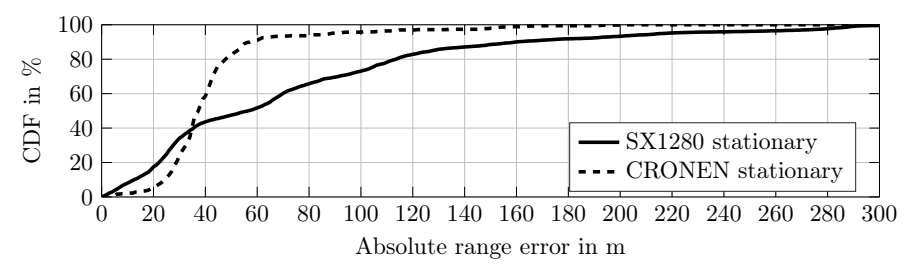

Fig. 12. LoRa $2.4 \mathrm{GHz} \mathrm{SX} 1280$ and CRONEN range error CDF for the urban canyon scenario.

Fig. 12 illustrates how CRONEN outperforms the SX1280 in the urban canyon.

\section{CONCLUSION}

Ranging benchmarking results for Narrowband LPWA technologies are compared in this paper (see Table II).

The LoRa $2.4 \mathrm{GHz}$ SX1280 radio chip allows obtaining sub-meter precision range estimates with a $1.6 \mathrm{MHz}$ signal bandwidth in AWGN channels. In a LoS ground based setup large range biases due to multipath reflections are observed and reduced with directional antennas. These findings strengthen the hypothesis that future accurate LPWA localization can be achieved by combining adequate ranging and beamforming technologies to reduce the impact of multipath channels.

In the comparative study between the LoRa $2.4 \mathrm{GHz}$ SX1280 radio chip and a coherent multi-channel ranging implementation for LPWA networks (CRONEN), both solutions reveal to be competitive. Performances are improved compared to existing TDoA based solutions, i.e. [6]. The SX1280 provides valid range estimations in mobile scenarios (speed $<36 \mathrm{~km} / \mathrm{h}$ ) while CRONEN fails. For stationary LoS and urban canyon scenarios

TABLE II

COMPARISION OF RANGING PERFORMANCES

\begin{tabular}{|c|c|c|c|c|c|c|}
\hline \multirow[b]{2}{*}{ Units in meter } & \multicolumn{3}{|c|}{ LoRa 2.4 GHz SX1280 } & \multicolumn{3}{|c|}{ CRONEN } \\
\hline & $\mu$ & $\sigma$ & $90 \%$ & $\mu$ & $\sigma$ & $90 \%$ \\
\hline $\begin{array}{l}\text { All measurements } \\
\text { Range } \leq 500 \mathrm{~m}\end{array}$ & 104 & 107 & 259 & 33 & 111 & 215 \\
\hline LoS moving & 60 & 61 & 143 & - & - & \\
\hline stationary & 75 & 69 & 145 & 32 & 63 & 116 \\
\hline NLOS stationary & 144 & 112 & 267 & 35 & 158 & 267 \\
\hline $\begin{array}{l}\text { Urban canyon } \\
\text { stationary }\end{array}$ & 70 & 70 & 159 & 36 & 34 & 57 \\
\hline
\end{tabular}

CRONEN outperforms the SX1280 due to its increased virtual bandwidth $\left(B W_{\text {virt }}=3 \mathrm{MHz}\right)$. Being able to scale ranging precision independently from receiver sensitivity, i.e. coverage is a strong argument for coherent multi-channel ranging. Transposing the CRONEN concept to the $2.4 \mathrm{GHz}$ ISM band offers a $80 \mathrm{MHz}$ bandwidth and excellent ranging precision.

Further investigation is needed on how NLoS propagation scenarios can be detected and excluded from the location solving process.

\section{ACKNOWLEDGMENT}

This work was supported by the European Union's Horizon 2020 research and innovation programme under 5G-HEART project (grant agreement No 857034).

\section{REFERENCES}

[1] 3GPP, "5G-HEART: 5G HEalth AquacultuRe and Transport validation trials," 2019.

[2] Link Labs, "LoRa Localization,"

[3] R. Ratasuk, N. Mangalvedhe, Y. Zhang, M. Robert, and J. P. Koskinen, "Overview of narrowband IoT in LTE Rel-13," in 2016 IEEE Conference on Standards for Communications and Networking (CSCN), Oct 2016, pp. $1-7$.

[4] X. Lin, J. Bergman, F. Gunnarsson, O. Liberg, S. M. Razavi, H. S. Razaghi, H. Rydn, and Y. Sui, "Positioning for the Internet of Things: A 3GPP Perspective," IEEE Communications Magazine, vol. 55, no. 12, pp. 179-185, Dec 2017.

[5] M. Aernouts, B. Bellekens, R. Berkvens, and M. Weyn, "A Comparison of Signal Strength Localization Methods with Sigfox," in 2018 15th Workshop on Positioning, Navigation and Communications (WPNC), Oct 2018, pp. 1-6.

[6] N. Podevijn, D. Plets, M. Aernouts, R. Berkvens, L. Martens, M. Weyn, and W. Joseph, "Experimental TDoA Localisation in Real Public LoRa Networks," in 2019 International Conference on Indoor Positioning and Indoor Navigation (IPIN), 2019.

[7] Semtech, "SX1280/SX1281 Long Range, Low Power, $2.4 \mathrm{GHz}$ Transceiver with Ranging Capability," 2017.

[8] M. Dogotari, "Hardware Design and RF Performance Evaluation of a Long Range 2.4 GHz Radio Module," Master's thesis, 2017.

[9] Wi6Labs, "Iot And Geo-Tracking : The Feedback Of A French Start-Up From Bretagne," 2018.

[10] S. Robinson, "Semtech SX1280 2.4GHz LoRa Ranging Tranceivers," https://github.com/LoRaTracker/SX1280_Testing, 2019, accessed: 2019/11/06.

[11] F. Wolf, J.-B. Dore, X. Popon, S. de Rivaz, F. Dehmas, and J. P. Cances, "Coherent Multi-Channel Ranging for Narrowband LPWAN: Simulation and Experimentation Results," in 15th Workshop on Positioning, Navigation and Communications (WPNC), October 2018, pp. 1-6.

[12] D. Vasisht, S. Kumar, and D. Katabi, "Decimeter-Level Localization with a Single WiFi Access Point," 2016.

[13] M. Pichler, S. Schwarzer, A. Stelzer, and M. Vossiek, "Multi-Channel Distance Measurement With IEEE 802.15.4 (ZigBee) Devices," IEEE Journal of Selected Topics in Signal Processing, vol. 3, no. 5, pp. 845-859, Oct 2009.

[14] A. Povalac and J. Sebesta, "Phase difference of arrival distance estimation for RFID tags in frequency domain," in 2011 IEEE International Conference on RFID-Technologies and Applications, Sept 2011, pp. $188-193$.

[15] Semtech, "Application Note: An Introduction to Ranging with the SX1280 Transceiver," March 2017.

[16] _ _ "Tools and Software: SX1280 Source Code PingPong and Ranging in C," 2017.

[17] M. Skolnik, Radar Handbook, 2nd ed. McGrawHill, 1990.

[18] O. Seller, "Ranging and Positioning System," EP Patent 2767 847, 2014.

[19] S. Marano, W. M. Gifford, H. Wymeersch, and M. Z. Win, "NLOS identification and mitigation for localization based on UWB experimental data," IEEE Journal on Selected Areas in Communications, vol. 28, no. 7, pp. 1026-1035, Sep. 2010. 